\title{
THE VISUAL PERCEPTION OF OBJECTIVE MOTION AND SUBJECTIVE MOVEMENT ${ }^{1}$
}

\author{
JAMES J. GIBSON
}

Cornell University

The perception of motion in the visual field, when recognized as a psychological problem instead of something self-evident, is often taken to present the same kind of problem as the perception of color or of form. Movement is thought to be simply one of the characteristics of an object, and the only question is "how do we see it?" Actually, the problem cuts across many of the unanswered questions of psychology, including those concerned with behavior. It involves at least three separable, but closely related problems: How do we see the motion of an object? How do we see the stability of the environment? How do we perceive ourselves as moving in a stable environment?

\section{Motion, Stability, and Movement}

The first problem concerns the visual perception of a moving object. It seems fairly simple as long as one considers a motionless eye. The stimulus condition for a moving object is the moving sheaf of light rays reflected from it. The retinal image accordingly moves relative to the retina and relative to the background image of the environment. The stimulus for visual movement is retinal

1 This research was supported in part by the United States Air Force under Contract No. AF33(038)-22373, monitored by the Perceptual and Motor Skills Research Laboratory, Human Resources Research Center Permission is granted for reproduction, translation, publication, use, and disposal in whole or in part by or for the United States Government. This report has been based on extended discussions. with and contributions from Donald M. Purdy. It also includes ideas and criticisms arising from collaboration with Olin W. Smith. movement. This definition is adequate, however, only for a fixated eye. It fails when we consider that the eye normally follows a moving object with a rotary pursuit movement that keeps the image of the object fairly precisely on the fovea. The background image then moves across the retina, but the object image does not. In this case the stimulus for the impression of motion is not so easy to define. A response is going on, and stimulation mediated by this response may enter into the picture. One might assume that movement of the object image relative to the background image but not the retina was the effective stimulus. Perhaps the observer senses the motion of the background and perceives the relative motion of the object. Or one might just as well assume that movement of the eye itself relative to the head or relative to the background inage but not the object image was the effective stimulus. Perhaps the observer senses the movement of the eye and thereby perceives the motion of the object. The alternatives are highly debatable, but for either one a difficult theoretical question arises: Why do we perceive a motion of the object in the environment instead of a motion of the environment? This leads to the second problem.

The second problem concerns the visual perception of a stable environment. Why does the world appear motionless, and what are the stimulus conditions for this perception? It is just as much a problem, if less obvious, as the first. Superficially considered, it appears simple for the case of the fixated eye: a motionless image yields a mo- 
tionless percept. It becomes difficult, however, for the case of the moving eye. Why does the phenomenal world not move during an eye movement? The eyes perform saccadic or exploratory movements without ceasing during waking life; they perform compensatory movements whenever the head moves; and they perform pursuit movements whenever a moving object catches the attention of the observer. Since the image of the environment moves across the retina during all these responses, the world should seem to move. It may be noted that with certain unusual types of eye movement an observer will report that the world does seem to move; examples are the after-nystagmus caused by bodily rotation (or other causes) and the artificial movement of the eye caused by pushing it with one's finger (11). During normal eye movements, however, the world does not seem to move, and this poses a question.

There are still other reasons for rejecting the simple hypothesis that a motionless image yields a motionless percept. They appear when we consider what happens when the obscrver moves.

The third problem concerns the visual perception of locomotion in a stable environment. We perceive not only the motions of objects but the movements of ourselves; the performance of fielding a baseball illustrates both. In the case of active locomotion, such as running, there is, of course, a large component of kinesthetic stimulation from the proprioceptors that accompanies the purely visual stimulation from the retinas. But in the case of passive or involuntary locomotion, such as riding in trains, automobiles, and planes, the kinesthetic component may almost wholly drop out. The visual component of stimulation results from the fact of motion parallax, and consists of differential motions of different parts of the image.
The writer and collaborators have recently given a mathematical description of this kind of stimulation for the general case of what is called motion perspective (8). The fact that it has to do with the perception of space has long been recognized, but the fact that it also has to do with the perception of locomotion is less well understood and deserves emphasis. The visual field during forward locomotion seems to expand radially from a point of focus on the line of locomotion. The optical geometry of this expansion is perfectly definite. The retinal image undergoes a deformation that can be neatly specified in terms of differential angular velocities. This retinal motion reaches high magnitudes during rapid travel, and there is reason to believe that it is the important factor in the performance of landing an aircraft. The apparent expansion of the visual field has been noticed by nearly everybody in driving an automobile. The question that arises is why the visual world does not seem to expand but instead seems to appear rigid, with the observer moving instead. The flier is never confused by the impression that his runway is behaving like stretched rubber.

It is worth noting that there are special cases of visual stimulation in which it does become equivocal whether the visual scene is moving or whether the observer himself is moving. If one sits looking through the window of a stationary railway train at another train on the adjacent track, and if one of the trains begins to move slowly, the impression of moving self with stationary scene may give way to that of stationary self with moving scene, or vice versa.

The three problems of the moving object, the stationary environment, and the moving observer are evidently interrelated. Objective motion is con- 
nected with subjective movement, ${ }^{2}$ since both stimulate the retina. The motion of an object, the movement of the eye, and the movement of the observer himself may alter the retinal image in different ways, but they all alter it. They are all inseparable from the problem of how or why we see the environment as stationary both when its image is altered and when it is stationary on the retina. One thing is clear at least: the kinetic experience in general involves the problems of so-called space perception.

Experimental Evidence on the PerCEPTION of Motion and MoveMrent

A survey of the established facts about the three problems may clarify them and even point to solutions. The experiments are not numerous, some of them are unfamiliar, and they have seldom been considered together.

\section{Motion of an Object}

Apparatus employed. Experimental studies of visual perception necessarily depend on devices for systematically

2 In this paper, for lack of a better terminology, the word motion will always be used to refer to change in position of an object, and the word movement will always refer to change in position of the observer's body in whole or part, that is, a response. Both may be visually perceived. The responses with which we are concerned are chiefly eye movements and locomotor movements. Movements of the limbs and hands are also important since they constitute a large part of behavior (gestures, manipulation, tool-using), and most of these are also visually perceived In them, however, the kinesthetic component, the muscle sense, is obviously important, and the visual component cannot be isolated for analysis as it can for locomotion. They are practically never passive or involuntary, as locomotion can be. They will not be considered bere. Nevertheless the writer believes that the visual feedback is just as important for motor performance as the bodily feedback, and that "visual kinesthesis" should be recognized along with classical kinesthesis. presenting light to the eyes of the observer, that is, methods of systematically varying his retinal images. In the case of motion, not many such devices have been successfully built. The types of apparatus for inducing controlled impressions of objective motion are approximately as follows: $(a)$ the stroboscope and the variants of this device, used to study apparent motion; $(b)$ the moving belt viewed through a window or aperture, used to study so-called "real" motion, or to induce the waterfall illusion; $(c)$ the rotating disk with a spiral, used to induce the impression of an expanding or contracting object and the negative afterimage of this impression; (d) the device of casting the shadow of a physically moving or rotating object on a translucent screen, the deforming shadow inducing the impression of a three-dimensional object in motion; (e) the device of rotating a disk with spiral lines behind a slotted screen, inducing the impression of objects moving along the slot. Practically all that is established about the perception of motion comes from one or another of these experimental methods. However, a novel device for presenting multiple complex motions on a translucent screen has recently been described by Johansson (12). One might suppose that the animated motion picture would have been used for controlled experimentation by psychologists, but it has scarcely been tried ( 5 , ch. 2). There have also been a number of setups with luminous spots in a darkroom, one or more of which are put into relative motion. This latter experiment, like the autokinetic illusion, is relevant to the problem of the stability of the environment as much as it is to the motion of an object.

Stroboscopic motion. The only large body of evidence based on these devices comes from the stroboscope. It is said to yield "apparent" motion as distin- 
guished from "real" motion, and the stroboscopic effect is often loosely referred to as the phi phenomenon. The stroboscope has evoked much research, probably because it demonstrates that a physically moving object is not necessary for an experience of motion, and because this seeming paradox has prompted psychologists to formulate controversial theories in order to explain it.

The important fact about stroboscopic motion, for present purposes, is that the stimulus is intermittent but that when certain relations hold, the perception of motion is the same as if the stimulus were not intermittent. As Troland asserted, "a perfect motion impression can be aroused without any actual motion of an object by the discontinuous substitution of one object for another at progressively different points in space" $(18$, p. 381$)$. This situation has frequently been reduced for experimental convenience to the case of tro successive light sources at two separated points in space. and this experiment has resulted in an elaborate Greekletter phenomenology of motion impressions (alpha, beta, gamma, delta, and phi). The results of this experiment have been reviewed elsewhere (for example, 1, ch. 15) and will not be discussed here. The fact is that when an adjacent order and a successive order of discrete stimuli are correlated, a continuous impression of an object in motion results. The main limitation seems to be that the interval between stimuli must not be too disproportionate to the separation between them. Hence stroboscopic stimulation differs from so-called "real" stimulation only in being discontinuous when the latter is continuous. The relations of order are the same in both.

Motion of a patterned surface. The speed and direction of linear motion are perceived with some accuracy when a moving belt is presented to the eye. The same thing is true for the rotary motion of the surface of a disk. For both, there are lower thresholds for velocity and also upper thresholds for velocity when motion turns into blur. Acuity for motion is high at the periphery of the retina considering how weak it is for color and form. There occurs a negative afterimage of velocity in a stationary visual field in that part of it which has previously been stimulated by a moving belt or disk. The afterimage may be linear or rotary or it may be one of expansion or contraction if the rotating disk bore a spiral that contracted or expanded (Plateau's spiral). The perceived velocity of a moving surface tends to be constant at different distances from the eye although the retinal velocity of its image is inversely proportional to distance. Brown, however, discovered some other puzzling facts about such apparent velocities connected with the size of the frame or aperture behind which the belt moved and with the brightness of the surface (2). Another fact, which is interesting for the problem of the connection between retinal motion and eye movement, is that perceived velocity is reported to be somewhat faster when the eyes are fixated on the aperture than when they follow the moving pattern from one side to the other and back again. This has been called the AubertFleischl paradox (2).

Deformation of shadows and the perception of depth. Linear and rotary motions presented to the eye by belts or disks occur in the frontal plane of the observer and are so perceived. So does the apparent expansion of a Plateau spiral, and this is also perceived as flat except for an occasional report that the afterimage suggests motion in depth. But the shadow of a rotating object observed from the other side of a translucent screen, although seen in one 
sense as moving in the frontal plane, is often seen in another sense as moving in depth. There may be a compelling impression of rigid rotation as well as an impression of deformation. This effect has been called stereokinetic, and Wallach has recently named it the kinetic depth effect (19). Metzger had previously studied the phenomenon and its interpretation (15). The impression of rotation in depth is reversible, and the observation of this feature of it goes back to "Sinsteden's windmill" ( 1 , p. 270).

Controllable complex movements. There have been a few experiments on multiple motions in the visual field, that is. of meaningless spots or shadows moving in systematically varied ways. Michotte, who used the method of a pair of rotated spirals visible through a horizontal slot in a screen, was interested in the perception of causality (16). Metzger, who projected on a translucent screen the shadows of vertical rods rotating on a horizontal turntable, was interested in the problem of the visual identity of the interpenetrating shadows (14). Johansson devised a method of superimposed slide projection in which each spot on the screen depends on a different slide and each slide can be given a controlled linear or circular motion. He was concerned with the perception of the events which his moving spots induced (12). Johansson also describes the other important experiments of this type. Heider and Simmel, using animated motion picture film, explored the possibilities of the social meanings which moving triangles and circles might evoke (10).

\section{The Stable Environment}

In contrast with the foregoing experiments in which the background of the motion, or the frame of the window in which it appears, is always visible stands a class of experiments utilizing points of light in a completely dark room. The case of a single fixated point has been studied for a long time. Although the image remains essentially motionless on the retina of the observer and the spot may appear at first to be static, it eventually shows an "autokinetic" motion. It appears to wander in an erratic fashion, and the observer himself may become disoriented. The illusion disappears if the surfaces of the room become even slightly visible. The facts are summarized by Carr (3, pp. 314 ff.). Evidently the stimulation of a single retinal point is not sufficient to yield the impression of a stable environment. Sandstrom has recently emphasized that an observer cannot even point with his finger to a single spot of light in a dark room (17). Facts of this sort throw great doubt on any kind of theory of the "local signs" of retinal points.

When two points of light are presented in the dark, their separation is sensed and they appear connected. They may appear to wander as an autokinetic unit, but one never appears to move relative to the other. It might be said that each has stability relative to the other.

If one of the two point sources in the darkroom is made to move slowly, the conditions are present for what Duncker has called "induced movement" (4). The observer reports motion, but it is as likely to be carried by the physically motionless source as by the moving source. A frequent outcome is a phenomenal motion of both spots, each carrying half of the total velocity. The relative motion of the first to the second or the second to the first (or each to the other) is perceptible, but the motion with reference to the room is not. The room, after all, is invisible and the background of the spots is darkness.

An example of induced motion taken from common experience is the appearance of the moon seen through drifting 
clouds. In this case the clouds provide an extended background for the moon, not just another spot of light, and the impression of the moon's motion is unequivocal. Duncker set up a similar situation and studied the apparent motion of a stationary spot of light projected on a rectangular surface that moved in pendular fashion from side to side. The relative motion of the spot within the frame was indistinguishable from "real" motion; it could be cancelled by setting up an opposite pendular motion of the spot itself (4).

Duncker also noted the occurrence of induced movement of the observer him. self, both in the darkroom situation and. under special conditions, with illumination. This was, of course, a movement without kinesthesis, produced wholly by visual stimulation. Insofar as an observer perceives himself in visual space, his own movement, like that of visual objects, depends on the phenomenal frame of reference. The question is, what establishes this frame of reference or stable visual environment?

\section{Movement of the Observer, Including Locomotion}

A simple method of inducing by visual stimulation one kind of apparent movement of the observer's body has long been known. It consists of surrounding the head of a stationary observer with a cylindrical screen or curtain, filling his entire visual field, which can then be rotated around the head. The observer reports a perception of being rotated in the opposite direction-an instance of Duncker's "induced ego-movement." The impression may be as vivid as that obtained from being actually rotated in a Barany chair, and the only difference between the case of rotating the miniature visual room and the case of rotating the observer may be the absence of vestibular stimulation in the former and its presence in the latter. The phenomenon is similar in principle to the "railroad train" illusion described earlier.

The analysis of motion perspective for a large portion of the visual field, also mentioned earlier ( 8 ), suggests that the impression of forward movement of the observer can be produced optically without any contribution from the vestibular or the muscle sense. This experiment, however, has not been performed. The closest approximation to it is an informal study based on a motion picture of the landing field ahead of an airplane during a glide $(5, \mathrm{p}$. 230). Observers reported an experience of locomotion along a glide path toward a visible spot on the ground. This perception was clearly, however, an "as if" kind of experience, pictorial rather than natural. The motion picture intercepted only a part of the field of view. It is said that the panoramic motion picture (especially the "Cinerama") induces even more compelling experiences of locomotion, such as a ride in a rollercoaster.

There has been little or no research on the contribution of kinesthetic, tactual, and vestibular sensitivity to the experience of passive locomotion. Their contribution to the sense of passive rotation of the body has been studied, and something is known about their contribution to the maintaining of upright posture. How kinesthesis is connected with the visually aroused impression of locomotion is not known. The flier and the automobile driver have muscular kinesthesis for the controls of the vehicle but not for the propulsion of the body, as in walking or running.

The experience of active locomotion -of voluntary or guided movement by the observer-is of course a still more complex psychological problem, which will not be touched on in this report. Most of the experimental evidence about 
voluntary action comes from studies of pursuit tasks, reaction time, and the like, which might be said to deal with manipulation rather than locomotion. A theory of movement with respect to a goal or destination is obviously of great importance, but we are here concerned with the cues or stimuli for movement as such. This may be justified on the grounds that the flow of actions, choices, or decisions during, for instance, an aircraft landing cannot be understood unless the flow of information is understood.

\section{Implications of the Evidence}

There is plenty of evidence to indicate that visual motion is a "sensory" variable of experience. It has a kind of intensity (speed) and a kind of quality (direction). It has absolute thresholds, both lower and upper, like pitch. Acuity depends on the part of the retina stimulated, like form. It has a negative afterimage, like hue. It tends to manifest constancy, like size and shape. In the form of "pure phi" it can be abstracted from an object. But more than any sensory impression, it fails to correspond to the physical stimulus presumed for it. Whatever the stimulus for motion might be, it is not simply motion in the retinal image. This seems to imply that motion is not sensory. Before concluding, however, that phenomenal motion is not a function of stimulation, the stimulus conditions should be re-examined.

The distinction between "real" and "apparent" motion is unfortunate and has interfered with the search for the essential conditions. It should be noted that stroboscopic stimulation can yield just as psychologically "real" a motion as does continuous stimulation, if certain relations are preserved. A stroboscope and a moving object are manifestly different, but they are the sources of stimulation, not the stimuli, and per- haps the latter are not so different after all. The facts of the experiments can be explained by the hypothesis that the retina responds to adjacent and successive order. If the orders correlate for the stroboscope and the object, the fact that the former is a discontinuous emitter may be unimportant. The two retinal images are similar in that the relations of order are the same in both; for example, right-left and before-after. The stimulus for motion, then, may be ordinal.

There is other evidence to suggest that the stimulus for motion is also relational. This means that it cannot be derived from the hypothetical "local signs" of retinal receptors. The fovea does not have a fixed value for breadth and height when stimulated by a single point of light. Moreover, as Duncker proved, the motion of one point of light on the retina is perceived relative to another point of light, not relative to the retina. The frame of reference for motion (or stability) seems to depend on the array of stimulation rather than the location of the receptors; it is transposable over the retina. Just as a motion for the physicist can be specified only in relation to a chosen coordinate system, so is a phenomenal motion relative to a phenomenal framework (13). Perceived motion occurs in a perceptually stable space or environment. Another way of saying this is to assert that the perception of stability is part and parcel of the perception of motion; you cannot have the latter without the former.

The optical stimulus conditions for a stable environment seem to be a retinal image containing many elements rather than a few or one. This can be described as a differentiated or "textured" image $(7,9)$. Perhaps stability goes with the perception of a surface or an array of surfaces extending over most of the field of view. The disappearance 
of the autokinetic illusion when the darkroom is even slightly illuminated is consistent with this hypothesis. So is the occurrence of the moon-in-the-clouds illusion. So also is the railroad train illusion when we take the window-filling train on the next track to be motionless. Perhaps the textural background image, whatever its relation to the anatomical retina, always tends to determine the phenomenal environment, and the more it approximates the total image the greater the stability. ${ }^{\mathrm{s}}$

Common experience suggests that we can perceive the motion of an object in depth as readily as its motion at right angles to the line of sight, and the experiments with deforming shadows on a translucent screen tend to bear out this suspicion. The kinetic depth effects so far obtained depend on perspective transformations of the shadows, and yield impressions of changing slant or rotation. There is no reason why they should not also be obtained with size transformations of shadows, which will yield impressions of linear approach and recession. A general hypothesis is suggested by these experiments, namely, that any regular transformation of a bidimensional image tends to yield a tridimensional motion in perception, and the kind of motion perceived depends on the kind of transformation. This hypothesis has the advantage of relating the experiments on moving shadows to experiments on shape constancy and size constancy, and suggests a principle of space perception that may be common to both. The fact that the transverse motions of a pair of belts observed at different distances can be judged equal in velocity when the surfaces are actually equal in velocity (if

3 This hypothesis is consistent with, if not essentially the same as, the position taken by Duncker in his admirable study of "induced" movement (4).
Brown's results [2] are accepted) points in the same direction.

Facts about the perception of bodily movement as distinguished from object motion are scarce. They are enough to suggest, however, that the impression of oneself being moved, like that of an object being moved, depends on the perception of the space in which the movement occurs. Ego movement like object movement can be induced. The train illusion and the cylinder rotating around the head are examples. The perception of forward locomotion can probably be induced, and the experiment should be tried. This will require optical stimulation governed by difierential angular velocities for many points in the visual field, i.e., motion perspective or, crudely speaking. an expanding image.

A promising bypothesis for research would be that any transformation of the total retinal image, as distinguished from a part image within it, tends to yield an expericnce of a movement of the observer, and the kind of movement experienced depends on the kind of transformation. For example, a simple translation of the image may contribute to the experience of an eye movement; an expansion may contribute to the experience of forward locomotion; a contraction to the experience of backward locomotion; and so forth.

There is said to be a striking lack of correspondence between the presumable optical stimuli and the ensuing visual perceptions of motion or movement. The evidence does indeed show what appear to be obvious discrepancies. It is certainly true that kinetic impressions are not copies of their stimuli. But it fails to follow that they are not functions of their stimuli. It cannot simply be assumed that a movement is the same thing in the object, the retina, the brain, and consciousness. The foregoing hypotheses make it possible to 
test for psychophysical correlations, although they do not imply any pictorial correspondence, between the dimensions of the stimulus and the qualities of kinetic experience.

\section{Hypotheses ABOUT KINETIC Retinal Strmulation}

A psychophysics of kinetic impressions would require a mathematical analysis and classification of the motions or transformations of a retinal image. This is a complex and difficult task for the future. Some preliminary assumptions are possible, however.

Geometrically, one can distinguish between a rigid and a nonrigid motion of a form or of a set of points. Translation and rotation are the types of rigid motion with which we are concerned. The figure after displacement is congruent or identical with the figure before displacement. The kinds of nonrigid motion are diverse and are still being explored by the higher branches of geometry. However, two classes exist, which may be called elastic motion and discontinuous or disjunctive motion. In the former, the lines of the geometrical form do not "break up" (or the set of points maintains the relations of neighborhood), whereas in the latter the form is ruptured (or the points are "scattered"). The class of elastic motions includes two types, the size transformations and perspective transformations on the one hand and nonperspective transformations on the other. The first type can be defined as a projection of the form or pattern on a plane different from its own, either an enlargement (or reduction) or a slant projection. The second type can be defined as a deformation other than these, but for which the continuity of the form is preserved. The class of disjunctive motions includes many types, which do not need to be specified here, but all involve discontinuity. The six types with which we are concerned are tabulated below:

\section{Rigid motion}

1. Translation

2. Rotation

\section{Elastic motion}

3. Size transformation

4. Perspective transformation

5. Deformation

\section{Disjunctive motion}

\section{Multiple movements}

These abstract mathematical motions are interestingly related to optical stimulation. Let us assume an eye and a reflecting surface, such as the face of an object toward the eye, and let us consider the cross-section of the sheaf of light rays to the nodal point of the eye (18, pp. 326 f.). This is equivalent to the retinal image. What tridimensional events produce these motions of the bidimensional cross-section? Numbers 1 and 2 above correspond respectively to a lateral movement of the eye (or the object) and a swivel movement of the eye (or a rotation of the object). Number 3 corresponds to a movement of the eye (or object) along the line between them. Number 4 corresponds to a planetary movement of the eye around the object or an inclination of the object to the line between them. Number 5 corresponds to an event confined to the object-a fluid or elastic motion of its substance. Finally, number 6 probably corresponds to an event such as the shattering of a single object or the interaction of multiple objects. Some of these statements need qualification in order to be exact, but they may serve as preliminary general rules. In other words, some very important types of physical events correspond to the geometrical types of motion in the projection. It is a reasonable hypothesis that the eye can register 
these geometrical types of motion when they occur in the retinal image.

It may have been noted that the physical events corresponding to motions number $1,2,3$, and perhaps 4 are ambiguous. Whether the eye moves or the object moves, the result is the same. The optical situation assumed in the previous paragraph consisted of an eye and a single object (specifically a plane face of an object). A more typical optical situation would consist of an eve and an environment. Let us therefore assume instead an eye and an infinite plane surface. This is a better approximation to the terrestrial environment. Except for the "sky," the image of the surface occupies the whole of the retina. and it constitutes a textured background image rather than a delimited object image. An infinite plane surface would be physically stable and would constitute an excellent frame of reference for visual perception. There is evidence to suggest that a background image does help to determine the stable phenomenal environment. Ambiguity of perception as to whether the eve moves or the environment moves in this situation would therefore tend to disappear."

The types of physical events producing the geometrical types of motion of a total background image are fairly univocal. Translation and rotation of this image can hardly be caused by anything but eye movements. Size and perspective transformations for the elements of an extended plane surface constitute motion perspective (8) and this can hardly be caused by anything but locomotion with respect to the surface. Certainly it is true that any eye movement in an illuminated environment

\footnotetext{
4 If to our disembodied eye we add assumptions about gravity, posture, muscles, and kinesthetic stimulation, the ambiguity would certainly disappear. But we are here concerned only with optical stimulation, admittedly an abstraction.
}

causes a rigid movement of the image, and any transportation of the eye causes an elastic movement of the image. $^{5}$

The causes in the environment and the results in perception of deformations and disjunctive motions of the image (numbers 5 and 6 above) are complicated. So far, we have been assuming a solid environment. Nonperspective deformations are caused by liquid or fluid motions of physical objects and surfaces. Rivers flow, smoke swirls, rubber stretches, and above all living organisms flex their surfaces in many ways. The faces of men, for instance, undergo an astonishing variety of rubbery motions, which we call facial expressions. We perceive these motions, sometimes with great acuity. We do not seem to confuse them with the mechanical motions of solid obiects which tilt, slant, advance, or recede with a kind of inanimate quality. There may be a basis in optical stimulation for this difference.

Disjunctive motions of the image are caused by a still greater variety of events. Objects break, ants swarm, billiard balls collide, and men shake hands. Michotte believes that multiple motions can yield immediate impressions of causation that are specific to the relations between them, and he has fortified his belief by experiments (16). The possibility of isolating high-order variables of stimulation in such images seems remote, but it should not be rejected.

In conclusion, the various motions of objects in a stable environment and the various movements of ourselves in that environment can both be visually perceived. A psychophysics of such kinetic impressions, however, is almost nonex-

- The classification of the motions of a retinal image here given is considerably revised from that proposed previously by the writer (6, p. 131 ff.). 
istent, and the possibility of isolating their stimuli has been doubted. If, however, the effective stimulation is taken to be ordinal and relational, it falls into several mathematical classes, which are neatly correlated with types of physical events, and which may prove to be psychophysically correlated with modes of kinetic experience.

\section{REFERENCES}

1. Borrvg, E. G Sensation and perception in the history of experimental psychology. New York: D. Appleton-Century, 1942.

2 Brows, J. F The visual perception of velocity Psychol. Forsch, 1931, 14, 199-232.

3. CARR, H. An introduction to space perception New York: Longmans, Green, 1935.

4 Duncker, $K$. Ober induzierte Bewegung Psychol Forsch., 1929, 12, 180-259.

5. Gibsox, J. J. (Ed.) Motion picture testing and research. Washington, D. C . Government Printing Office, 1947. (AAF Aviat. Psychol. Program Res. Rep. No. 7.)

6. Grison. J. J. The perception of the visual world Boston: Houghton Mifflin, 1951.

7 Gibson, J. J., \& Dibble, F. N. Exploratory experiments on the stimulus conditions for the perception of a visual surface. J. exp. Psychol., 1952, 43, 414-419.

8. Gibson, J. J , Olum, P., \& Rosenblatt, F. Motion parallax and motion per- spective in aircraft landings. $A F$ Hum Resour. Res. Cent. Bull., in press

9. Gibson, J. J., \& Waddell, D. Homogeneous retinal stimulation and visual perception Amer. J. Psychol., 1952, 65 , 263-270.

10 HeIder, F., \& StMmel, $M$ An exper1mental study of apparent behavior Amer. J Psychol., 1944, 57, 243-259.

11 HoLT, E B Eye-movement and central anaesthesia. Psychol. Monogr., 1903. 4, No. 1 (Whole No. 17), 3-46.

12. Johansson, G. Configurations in event perception. Uppsala: Almqvist \& Wibsell, 1950.

13. Korfra, K. Principles of Gestalt psy. chology. New York: Harcourt, Brace, 1935.

14. Metzger, W. Beobachtungen über phänomenale Identitat. Psychol. Forsch, 1934, 19, 1-60.

15. MetzGer, W. Tiefenerscheinungen in optischen Bewegungsfeldern Psychol Forsch., 1935, 20, 195-260.

16 Michotтe, A La perceplion de lo causalité. Louvain: Inst. sup. de Philosophie, 1946.

17. SANDSTRom, C. I. Orientation in the present space Uppsala: Almqvist \& Wiksell, 1951.

18 Troland, L. T. Principles of psychophysiology. Vol 1. Problems of psychology and perception. New York: Van Nostrand, 1929.

19 Wallace, H., \& O'Conneil, D. N. The kinetic depth effect J. exp Psychol., 1953, 45, 205-217.

(Received November 16, 1953) 